\title{
Reflets
}

Revue ontaroise d'intervention sociale et communautaire

Miriam Ticoll, La Violence et les personnes ayant des incapacités : une analyse de la littérature, Institut Roeher Institute pour le Centre national d'information sur la violence dans la famille, Santé Canada, mars 1995, 93 p.

\section{Richard Carrière}

Volume 4, numéro 2, automne 1998

Personnes vivant avec une incapacité

URI : https://id.erudit.org/iderudit/026242ar

DOI : https://doi.org/10.7202/026242ar

Aller au sommaire du numéro

Éditeur(s)

Reflets : Revue ontaroise d'intervention sociale et communautaire

ISSN

1203-4576 (imprimé)

1712-8498 (numérique)

Découvrir la revue

Citer ce compte rendu

Carrière, R. (1998). Compte rendu de [Miriam Ticoll, La Violence et les personnes ayant des incapacités : une analyse de la littérature, Institut Roeher Institute pour le Centre national d'information sur la violence dans la famille, Santé Canada, mars 1995, 93 p.] Reflets, 4(2), 235-238.

https://doi.org/10.7202/026242ar

Tous droits réservés (C) Reflets : Revue ontaroise d'intervention sociale et communautaire, 1998
Ce document est protégé par la loi sur le droit d'auteur. L'utilisation des services d’Érudit (y compris la reproduction) est assujettie à sa politique d'utilisation que vous pouvez consulter en ligne.

https://apropos.erudit.org/fr/usagers/politique-dutilisation/ 


\section{La Violence et les personnes ayant des incapacités : une analyse de la littérature}

TICOLL, MIRIAM. Institut Roeher Institute pour le Centre national d'information sur la violence dans la famille, Santé Canada, mars 1995, $93 \mathrm{p}$.

par

Richard Carrière

École de service social, Université Laurentienne

Depuis les trente dernières années, la maltraitance des enfants, des femmes et des personnes âgées est un phénomène qui a fait couler beaucoup d'encre tant chez les professionnels que dans le grand public. Si nos bibliothèques débordent d'études portant sur la violence qui s'exerce contre ces groupes, «les textes consacrés à la violence faite aux personnes ayant des incapacités [...] sont relativement peu nombreux». C'est du moins la conclusion à laquelle parvient Miriam Ticoll, de l'Institut Roeher, après avoir élaboré une bibliographie sur le sujet.

Ce texte est la traduction française d'un document intitulé, Violence and People with Disabilities: A Review of the literature. Par conséquent, la plupart des études citées sont anglaises, provenant du Canada, des États-Unis ou de la Grande-Bretagne. Un des auteurs les plus souvent répertoriés est Dick Sobsey qui, en 1994, a publié un livre intitulé, Violence and Abuse in the Lives of People With Disabilities: The End of Silent Acceptance. Les quelques références françaises sont surtout des traductions provenant d'organismes fédéraux. Ainsi, on ne trouve au total, malheureusement, que 35 documents en français dans une bibliographie exhaustive comportant 32 pages.Vous remarquerez également dans la bibliographie soumise par Lorraine Albert pour ce numéro de la revue Reflets, l'absence de texte français portant sur la violence subie par les personnes ayant une incapacité. 
Le texte de Miriam Ticoll de l'Institut Roeher offre donc un des rares documents en français permettant de connaitre le contexte particulier de la violence infligée aux personnes vivant une incapacité. Il est très bien organisé. Dans un premier temps, l'auteure résume le sujet en dégageant ses principaux éléments. Mais, ne vous arrêtez surtout pas là! En effet, la lecture complète du texte se veut des plus intéressantes.

Dans son introduction, Miriam Ticoll décrit en détail la méthodologie utilisée pour effectuer sa recherche bibliographique. On découvre ainsi qu'elle a utilisé des banques de données sur cédérom ou Internet comme Psych Lit, Sociofile et Abstracts in Criminology and Penology. On peut d'ailleurs le lui reprocher car, selon le Cochrane Institute, un organisme international spécialisé dans la recension systématique des écrits scientifiques, les banques de données sur cédérom ou Internet permettent d'identifier environ $40 \%$ des écrits portant sur un sujet de ce genre. En effet, plusieurs petites revues spécialisées et les rapports d'organismes privés et nongouvernementaux ne sont pas répertoriés dans ces grandes banques de données. Cela dit, l'auteure a élaboré une bibliographie fort adéquate, bien qu'en la parcourant, on se rende vite compte du peu d'études canadiennes portant sur la problématique en question.

En plus du sommaire et du premier chapitre introductif, le rapport comporte sept autres chapitres, suivis d'une conclusion et d'une bibliographie. Le deuxième chapitre porte sur la définition de la violence faite aux personnes ayant des incapacités. Il est important car il aide à comprendre que si les personnes ayant des incapacités sont victimes des mêmes formes de violence que le reste de la population, certaines d'entre elles sont encore plus vulnérables à certains types de mauvais traitements. L'auteure avance que «la détermination des diverses formes de violence faite aux personnes ayant des incapacités dépend dans une certaine mesure de l'inclusion ou non de la discrimination systémique et de d'autres formes de violence qui ne sont pas nécessairement des infractions criminelles» (1995:10).Ainsi,Ticoll présente de nombreux exemples de violence susceptibles d'être vécus par des personnes ayant une incapacité : les électrochocs, les techniques de coercition pour la modification du comportement ou encore certains traitements 
expérimentaux. Elle mentionne également que «les recherches récentes confirment le point de vue selon lequel les menaces, les insultes et le harcèlement sont des constantes dans la vie de nombreuses personnes ayant des incapacités» $(1995: 14)$.

Dans le troisième chapitre, l'auteure souligne qu'il est difficile d'obtenir un portrait clair de l'ampleur de la violence faite aux personnes ayant une incapacité, car trop souvent, même en s'en tenant à une définition légale, les statistiques officielles ne distinguent pas si la victime a une incapacité ou non. Les expériences des populations marginalisées sont ainsi reléguées à l'invisibilité. Toutefois, à partir des quelques études qui ont tenté d'identifier les victimes ayant une incapacité,Ticoll présente un tableau des formes de violence, des victimes et du pourcentage de victimes ayant une incapacité. Ces études suggèrent que les personnes vivant une incapacité ont un risque plus élevé d'être victimes de violence.

Le quatrième chapitre examine les milieux familial et institutionnel où se produit la violence. En lien avec toute la discussion contemporaine sur ce qu'est la famille, l'auteure démontre que «dans la littérature sur l'incapacité, le mot famille signifie non seulement parents, époux, amis de coeur et autres membres de la famille mais également les amis, les voisins et les préposés aux soins» (1995 : 24). Il s'agit donc de gens dont dépendent les personnes ayant une incapacité pour leurs besoins quotidiens. Même si l'auteure inclut les pourvoyeurs de soins dans sa définition d'une famille élargie, elle examine de façon particulière la violence au sein des établissements de services, car «le grand nombre de personnes concernées, le contact intime, physique et affectif nécessaire pour assurer les soins et le déséquilibre de pouvoir entre le prestataire et le bénéficiaire des soins sont des facteurs qui accroissent les risques de violence» (1995: 27).

Le prochain chapitre porte sur les éléments généraux et particuliers contribuant à la vulnérabilité des personnes ayant des incapacités. Comme le soulignent plusieurs chercheurs, «ce n'est pas l'incapacité à proprement parler qui rend les personnes vulnérables mais les conditions sociales dans lesquelles elles se trouvent»(1995: 32). Une liste des principaux facteurs de risque est présentée. Ce chapitre est très bref et aurait profité d'une 
discussion plus approfondie des facteurs énumérés. Pour établir un lien avec les chapitres précédents, il aurait été important de discuter de ce que David Gil (1970), dans son étude sur les enfants maltraités, a nommé le niveau sociétal de la violence. Gil faisait référence aux politiques et aux programmes sociaux qui précipitent les enfants dans des circonstances néfastes à leur bien-être. En raison du désengagement progressif de l'État, il aurait été important de discuter des différents facteurs à la lumière de cette violence sociétale, car plusieurs personnes vivant avec des incapacités n'ont pas le choix des conditions sociales dans lesquelles elles se trouvent. Par exemple, lorsqu'on réduit les budgets alloués aux services de transport, on risque d'isoler davantage les personnes. Or, nous savons que l'isolement social est un facteur important dans les dynamiques sociales de la violence.

Les chapitres six et sept sont eux aussi très courts, en raison du peu de recherches portant sur leurs thèmes respectifs, soit les effets de la violence et la révélation de la violence par les victimes. Le huitième chapitre examine les écrits sur les réactions de la police, du système judiciaire, des établissements de services et des services de soutien communautaires face à la violence subie par les personnes ayant des incapacités.

En conclusion, l'auteure reprend certaines suggestions faites par divers auteurs ayant trait à la prévention de la violence infligée aux personnes vivant une incapacité. Contrairement aux travailleuses sociales et aux travailleurs sociaux, elle n'utilise pas les concepts de prévention primaire, secondaire ou tertiaire. Elle regroupe plutôt ses remarques sous les thèmes des changements systémiques, des mesures préventives dans divers milieux et des réponses aux incidences de mauvais traitements.

Toutes les intervenantes et tous les intervenants œuvrant dans les domaines sociaux et communautaires bénéficieraient de la lecture de ce texte. On peut d'ailleurs se le procurer gratuitement en appelant le Centre national d'information sur la violence dans la famille à Ottawa ${ }^{1}$. Miriam Ticoll et l'Institut Roeher doivent être félicités pour la qualité de leur analyse.

1. 1-800-267-1291 